\title{
Study on influence of air decouple charge impact on blasting stress field in rock
}

\author{
Q Zong \& H B Wang \\ School of Civil Engineering and Architecture, Anhui University of Science and Technology, \\ Huainan, Anhui 232001
}

KEYWORD: Air; Decoupled charge; Blasting stress field; Experimental Investigation ABSTRACT: According to the theory of detonation and stress wave, initial shock pressure in wall of air-decoupled charge was deduced in theoretical and blasting stress field of air-decoupled charge in rock was established. Influence of decoupled coefficient to blasting stress field was analyses combined with test result, the result further proved decoupled charge can reduce compression stress obviously, the greater coefficient and stress reduce the more notable.

\section{INTRODUCTION}

At present, decoupled charge structure is one of widespread used charge structure; air and water are commonly used coupling medium, for the detonation wave and gas indirectly impact on blast-hole wall by through couplant, so compression force and blasting stress field in are different for difference between couplant. Both home and abroad also carried out many research in this area, mainly concentrated in blasting energy propagation of water and air decoupled, initial shock pressure, experimental investigation and numerical simulation of blasting mechanism ${ }^{[1 \sim 7]}$. However, theoretical study of air decoupled charge blasting stress field are seldom find, this article intended calculated blasting stress field of air-coupled charge in theoretical, and combined with the test results analysis influence of decoupled coefficient on blasting stress field.

\section{BLASTING STRESS FIELD CALCULATION OF AIR DECOUPLE CHARGE}

\section{CALCULATION OF HOLE WALL INITIAL SHOCK PRESSURE}

There are multiple calculation methods for air decoupled charge blasting hole wall the initial shock pressure calculation, basically have the following several:

1) Assumptions no air exist in the gap, explosion detonation products entropy expansion in gap with $k=3$, expansion initial pressure is average explosion detonation pressure, so evaluated hole wall initial impact pressure as follow ${ }^{[8]}$ :

$$
P_{b}=n P_{1}=\frac{n \rho_{e} D_{V}^{2}}{2(k+1)} \times K_{d}^{-2 k}
$$

Where: $P_{1}$ is explosion detonation products incident pressure before collision hole wall; $\rho_{e}$ is density of explosives; $D_{V}$ is detonation velocity of explosives; $K_{d}$ is decoupled coefficient of hole, $K_{d}=d_{b} / d_{c}, d_{b} 、 d_{c}$ is diameter of blasting hole diameter and charge respectively; $n$ is multiple of pressure increased when detonation gas impact hole wall, according to reference[8], $\mathrm{n}=8 \sim 11$.

2) Air exists between charge and hole wall, air shock wave generates in gap at first after explosive blast, then air shock wave impact on hole wall causes hole pressure, its value is:

$$
P_{b}=\frac{2 n}{\bar{k}+1} \times \rho_{a} D_{a}^{2}
$$

Where, $\rho_{a}$ is air density $\left(\rho_{a}=1.25 \times 10^{-3} \mathrm{~g} / \mathrm{cm}^{3}\right) ; D_{a}$ is air shock wave propagation velocity in hole wall, and related with charge decoupled coefficient and detonation products diffusion speed; $\bar{k}$ is average adiabatic index of air in gap, $\bar{k}=1.17 \sim 1.25 ; n$ still is multiple of pressure increased when detonation gas impact hole wall, but its value is different from preceding, $n=0 \sim 20$, exact value depends on incidence of air shock wave pressure. 
3) Above two methods are calculated by one-stage constant entropy expansion, which is different from the actual detonation gas state, especially at larger charge decoupled coefficient. So some studies suggest that, expansion process of detonation products should be considered as two-stage constant entropy expansion ${ }^{[9 \sim 11]}$, for average power industrial explosives, critical pressure of twostage demarcation point $P_{K}=200 \mathrm{MPa}$. When incidence of air shock wave pressure $P_{1} \geq P_{K}$, When incidence of air shock wave pressure $P_{1} \geq P_{K}$, calculated in accordance with k=3 constant entropy expansion, when $P_{1}<P_{K}$, calculated according to the $\gamma=1.3$ adiabatic expansion, so:

When, $\mathrm{P}_{1} \geq P_{K}$

$$
P_{b}=\frac{n \rho_{e} D_{V}^{2}}{2(k+1)} \times\left(\frac{d_{c}}{d_{b}+x}\right)^{2 k}
$$

When, $P_{1}<P_{K}$

$$
P_{b}=\left(\frac{P_{w}}{P_{K}}\right)^{\gamma / k} \times n P_{K} \times\left(\frac{d_{c}}{d_{b}+x}\right)^{2 \gamma}
$$

Where, $P_{1}$ is explosion detonation products incident pressure before collision hole wall; $P_{K}$ is critical pressure of two-stage demarcation point, its value is $200 \mathrm{MPa} ; n$ is multiple of pressure increased when detonation gas impact hole wall, general values is $8 ; \rho_{e}$ is density of explosive; $D_{V}$ is detonation velocity of explosive; $k$ is adiabatic index of detonation gas, $k=3 ; d_{b} 、 d_{c}$ is diameter of blasting hole diameter and charge respectively; $x$ is an instantaneous hole wall displacement; $\gamma$ is entropy of ideal gas, $\gamma=1.3 ; P_{w}$ is average detonation pressure, its value:

$$
P_{w}=\frac{\rho_{e} D_{V}^{2}}{2(k+1)}
$$

4) Because hole wall displacement is too small for negligible, in this condition, derived the following initial hole wall shock pressure formula of decoupled charge blasting ${ }^{[12]}$ :

$$
P_{r}=P_{d} \times K_{d}^{-2 k}=\frac{\rho_{e} D_{e}^{e}}{k+1} \times \frac{2 \rho_{m} C_{p}}{\rho_{m} C_{p}+\rho_{e} D_{e}} \times K_{d}^{-2 k}
$$

Physical meaning of symbol in the equation is the same as former. The formula adequately take influence of rock properties into consider, this is fundamental different from the above calculation method. In fact, extent of incident wave refraction and reflection at the hole wall is different for rock's impedance diversity.

\section{BLAST STRESS FIELD CALCULATION}

Put hole wall of the initial shock pressure of air-decouple charge into stress wave attenuation formula $^{[8]}$, access to blasting stress field of air-decoupled charge, that is,

$$
\sigma_{r}=\frac{P_{r}}{r}=\frac{p_{d} \times K_{d}^{-2 k}}{r}=\frac{\rho_{e} D_{e}^{e}}{k+1} \times \frac{2 \rho_{m} C_{p}}{\rho_{m} C_{p}+\rho_{e} D_{e}} \times K_{d}^{-2 k} \times\left(\frac{r}{r_{b}}\right)^{-\alpha}
$$

\section{BLASTING MODEL EXPERIMENT AND RESULTS ANALYSIS}

According to similarity theory and similarity criterion, small charge concrete model blasting experiment were did, tested the explosion stress in specimen, research the influence of decoupled coefficient to blasting stress field.

\section{MODEL PRODUCTION AND TESTING SYSTEM}

Model is poured by $425^{\#}$ Portland cement with sifted sand. To reduce the influence of boundary effect, we use rather larger sizes of model $350 \mathrm{~mm} \times 450 \mathrm{~mm} \times 700 \mathrm{~mm}$, maintenance more than 28 days. Strain brick use the same material and ratio as the test block, sizes is $30 \mathrm{~mm} \times 30 \mathrm{~mm} \times 300 \mathrm{~mm}$. Polish with sandpaper until it is smooth, then stick the strain gauge (BX120-5AA) with glue 502, its sensitivity coefficients is $2.08 \%$ and resistance $120 \Omega .8$ pieces for each strain brick, and layout a point every $60 \mathrm{~mm}, 4$ points totally. We welding on the single cable after the strain gages are sticked, insulating and waterproofing it with $\mathrm{AB}$ glue. 
Making use of the pre-receive strain brick as sensor to receive explosion signal, using super dynamic strain indicator and oscilloscope to collect and analyze the data. The super dynamic strain indicator are manufactured by Institute of Mechanics, Chinese Academy of Sciences, and the oscilloscope were manufactured by Agilent Technologies of United States, and the model number is 54815A, with bandwidth $500 \mathrm{MHz}, 4$ channels, sampling rate $1 \mathrm{GSa} / \mathrm{s}$.

Drilling fixed depth as $180 \mathrm{~mm}$ during the experiments, using special electric detonators (diameter of the detonator is $6 \mathrm{~mm}$, length of $40 \mathrm{~mm}$, installed $0.3 \mathrm{~g}$ dinitrodiazophenol, charge density of 1.0 $\mathrm{g} / \mathrm{cm}^{3}$, detonation velocity $4200 \mathrm{~m} / \mathrm{s}$, explosion heat $4000 \mathrm{~kJ} / \mathrm{kg}$ ). Small detonator installed to the end of hole, reserved air in the hole, and blocked off with fine sand and 502 glue, length of 40mm.

\section{Test results and analysis}

Test results shows in Table 1.

Table 1 Actual measurement results of radial stress

\begin{tabular}{cccccc}
\hline Hole diameter & Decoupled & \multicolumn{4}{c}{ Stress (MPa) } \\
\cline { 3 - 6 }$(\mathrm{mm})$ & coefficient & 1 & 2 & 3 & 4 \\
\hline 10 & 1.67 & 112.13 & 94.137 & 66.268 & 23.008 \\
12 & 2.00 & 99.089 & 75.716 & 41.618 & 16.918 \\
14 & 2.33 & 89.072 & 50.151 & 35.172 & 10.586 \\
16 & 2.67 & 61.424 & 47.506 & 13.429 & 7.105 \\
\hline
\end{tabular}

Curves of peak stress with proportional distance of different decoupled coefficient as Figure 1 and Figure2 shows; take testing data into formula (7), curves of calculated peak press with proportional distance as show in Figure3 and Figure4.

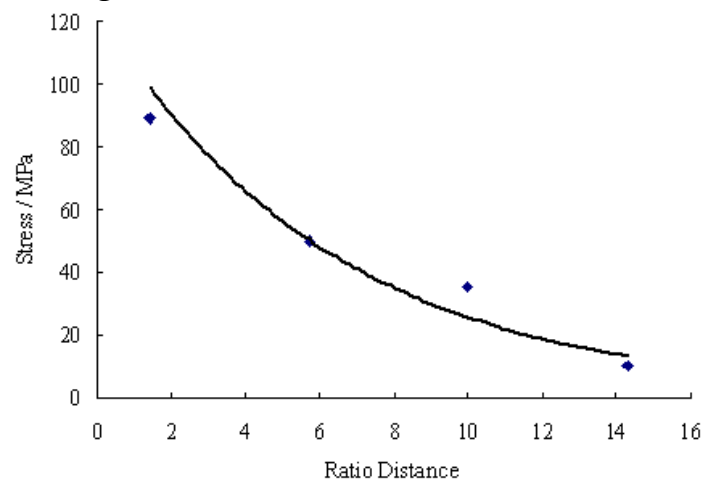

Figure1 The curve of peak pressure along with ratio distance $\left(K_{d}=2.33\right)$

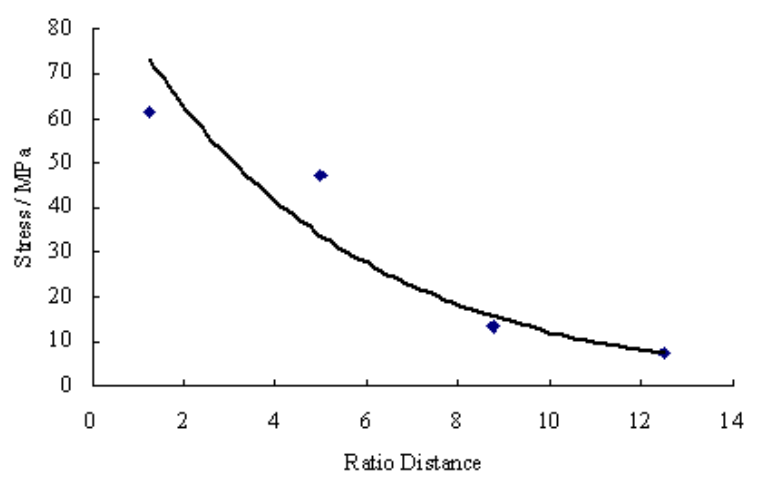

Figure2 The curve of peak pressure along with ratio distance $\left(K_{d}=2.67\right)$

According to testing and calculated results in Table 1, attenuation law of peak stress value with distance of air-decoupled charge blasting is: 


$$
\begin{aligned}
& K_{d}=1.67, \quad \sigma_{r}=295.83 \bar{R}^{-0.5524} \\
& K_{d}=2.00, \sigma_{r}=271.7 \bar{R}^{-0.664} \\
& K_{d}=2.33, \quad \sigma_{r}=243.15 \bar{R}^{-0.7779} \\
& K_{d}=2.67, \quad \sigma_{r}=182.52 \bar{R}^{-0.8915}
\end{aligned}
$$

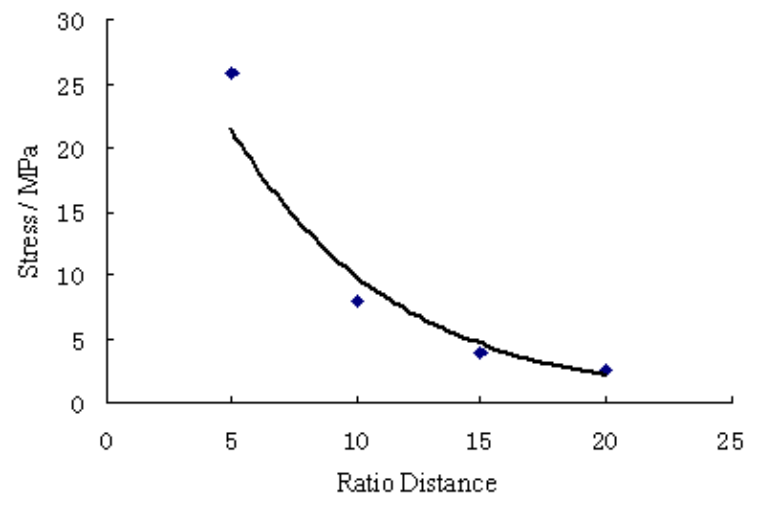

Figure3 The curve of stress along with ratio distance $\left(K_{d}=2.33\right)$

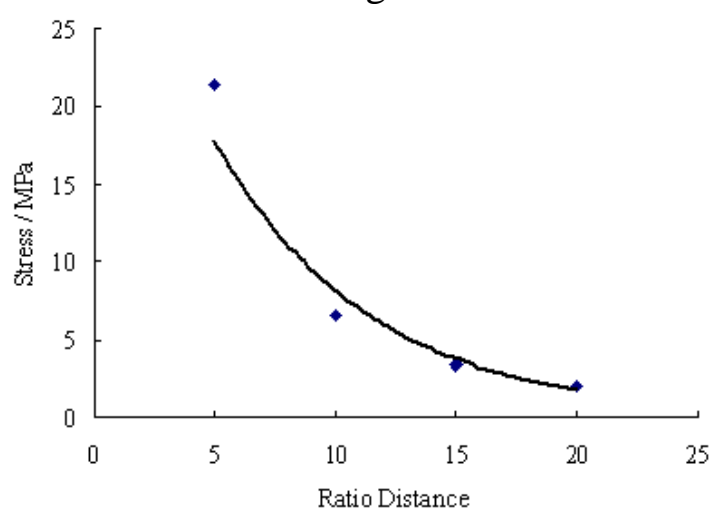

Figure4 The curve of stress along with ratio distance $\left(K_{d}=2.67\right)$

From blasting model test results and analysis conclusions can draw:

(1) The more near to the hole, the higher explosion stress, the peak stress decreased significantly as the distance increases, and decreasing rate of initial phase is larger, slow decline after the next stage, stress "platform phenomenon" appeared.

(2) With increment of decoupled coefficient $K_{d}$, the same measurement point's peak stress decreased, reaction time increased. With the increase of $K_{d}$ reflects explosives dynamic and static action relative change, its dynamic action weakening, static action strengthening. This is further proved the charge decouple coefficient does have significant influence on blasting pressure, air in the gap played a buffer role, The first explosive detonation wave compressed couple medium in the gap, forming a thin class of solid-phase material, a lot of elastic energy collected, so that part of the kinetic energy inverted, peak pressure caused by initial shock effect decreases and then, collected elastic energy released through the detonation gas, resulting "platform phenomenon", thus delaying the pressure attenuation, extended duration of action.

\section{CONCLUSIONS}

In this paper, blasting stress field calculation formula was deduced in theoretical, and influence of decoupled coefficient to blasting stress field was analyses combined with test result. The result proved that decoupled charge can reduce compression stress obviously, the greater coefficient and stress reduce the more notable. In actual blasting engineering, should according to engineering re- 
quirements, rock and construction conditions, choose rational charging structure, such as choose decouple charge structure with bigger decouple coefficient for presplit blasting, smooth, etc.

\section{ACKNOWLEDGEMENTS}

This research was financially supported by the National Natural Science Foundation (Grant No.51404010, 51374012, 51274009) and the Key Scientific and Technical Program of Anhui Province (No: 1501041123). The authors are grateful to the reviews for their valuable comments.

\section{REFERENCES}

[1] Zong Qi. Theoretical Analysis of Charge Structure's Influence on Pressure on Bore Wall [J]. Mining and Metallurgical Engineering, 2006, 26 (5): 9-13.

[2] Zong Qi, Luo Qiang. Experimental Study on Distribution Character of Blasting Stress When Boreholes with Water-Couple Charge [J]. Journal of Experimental Mechanics [J]. 2006, 21 (3): 393-397.

[3] Zong Qi, Meng Dejun. Influence of Different Kinds of Hole Charging Structure on Explosion Energy Transmission [J]. Chinese Journal of Rock Mechanics and Engineering, 2003, 22 (4): 641645.

[4] Du Junlin, Zhou Shengbing, Zong Qi. Theoretical analysis on pressure on bore wall of uncouple charge [J]. Journal of Xi ' an University of Science and Technology, 2007, 27 (3): 347-351.

[5] Wu Liang, Wei Min, Zhong Dongwang, et al.. Study on dynamic stress characteristics of airdecked charge explosion [J]. Blasting, 2009, 26 (4): 17-21.

[6] Wu Haijun, Yang Jun, HUANG Fenglei, et al.. Propagation behavior of stress wave of rock under the conditions of different coupling charges [J]. Mining Research and Development, 2002, 22 (1): 44-47.

[7] Jiang Pengfei, Tang Degao, Long Yuan. Numerical analysis of influence of uncoupled explosive-charge structure on stress field in hard rocks [J]. Rock and Soil Mechanics, 2009, 30 (1): 275279.

[8] Xu Ying, Zong Qi. Theory and Application of Underground Blasting [M]. Xuzhou: China Mining University Press, 2001.

[9] Gao Jinshi, Zhang Qi. Blasting Theory and Optimization[M]. Xi'an: Xi'an Map Press, 1993.

[10] Fei Honglu, Li Shouju, He Qingzhi. Determination of Decouple Coefficient and Analysis of Decouple Action in the Smooth Blasting. Explosion and Shock Waves, 1992, 12(3): 270-273.

[11] J.Henrych; Xiong Jianguo, et al.(Translation). The Dynamics of Explosion and Its Use [M]. Beijing: Science Press, 1987.

[12] Zhu Ruigeng, Wang Xuefeng, Decouple Charge Blasting Hole Wall Pressure Calculation [J]. Blasting, 1990, 7(3):1-3. 